\title{
SOCIEDAD CIVIL Y GOBERNANZA DE LA CIENCIA Y LA TECNOLOGÍA EN EL ÁMBITO AUTONÓMICO: LA COMUNIDAD AUTÓNOMA DE MADRID 1
}

\author{
Betty Estévez Cedeño \\ Departamento de Ciencia, Tecnologia y Sociedad \\ Instituto de Filosofia, CSIC
}

\begin{abstract}
Nowadays, the plurality of actors in the design of science and technology policy is a significant subject. Therefore, the promotion of public participation becomes an important challenge for the national and regional administrations. The main contribution of this paper is the analysis and assessment of citizen participation opportunities and difficulties at the regional level, in order to generate science policies better related to social needs. To begin with, we have analysed the relevance of citizen participation in the governance of science and technology. The results of the empirical work carried out in the Autonomous Community of Madrid demonstrate that a growing number of actors consider positively the involvement of "social experts" in the negotiation and design of regional science and technology programs. The need to activate strategies for the interaction between scientists and citizens has also been identified, especially in high-priority areas.
\end{abstract}

KEY WORDS Science and technology policy, science democratization, citizen participation, governance.
RESUMEN La necesidad de ampliar la diversidad de actores en el diseño de la política científica y tecnológica es un asunto cada vez más acuciante. Por tanto, la incorporación de la participación ciudadana en la orientación y diseño de estas áreas se convierte en un reto para las administraciones nacionales y regionales, principalmente. El aporte primordial de este trabajo es evaluar las posibilidades y dificultades de la participación pública en el diseño de planes regionales de ciencia y tecnología como una acción significativa para generar propuestas más acordes con la realidad social. Como punto de partida se considera que la participación ciudadana está presente en la nueva noción de gobernanza de la ciencia y la tecnología. Los resultados del trabajo empírico realizado en la Comunidad de Madrid entre los actores que participaron en la elaboración del IV Plan Regional de Investigación Científica e Innovación Tecnológica evidencian que un importante número de actores se inclina por la idea de que la implicación de representantes de base comunitaria o expertos sociales es positiva para moldear programas científico-técnicos. También se constata la necesidad de precisar y poner marcha estrategias de interrelación entre comunidad científica y sociedad según las áreas consideradas como prioritarias.

PALABRAS CLAVE Política científico-tecnológica, democratización de la ciencia y la tecnología, participación ciudadana, gobernanza.

\section{Introducción}

Las nuevas orientaciones de la administración europea dan cuenta de los cambios surgidos en muchas de las instituciones que constituyen la sociedad comunitaria del siglo XXI. En el ámbito de la ciencia y la tecnología, la Unión Europea apoya la promoción de nuevas formas de hacer políticas basadas en la ampliación del intercambio entre los niveles de gobierno (trasnacional, nacional y regional), el fortalecimiento de la participación de los actores involucrados y/o afectados, y la integración del conocimiento generado a partir de su interrelación (Banthien et al, 2003; Lebessis et al, 2000; Elam et al, 2003;
Edler et al, 2003). La relación entre estos factores se formaliza a través de instituciones que participan en un nuevo tipo de juego multinivel canalizado por estrategias especificas.

Los cambios plantean que la nueva forma de hacer política científica y tecnológica necesita de la presencia y participación acentuada de la ciudadanía. Argumentar sobre esta premisa constituye la base fundamental de este trabajo. Se hace énfasis en la significación de la participación pública en el diseño de planes regionales de ciencia y tecnología. La tesis subyacente es que la participación ciudadana está presente en la composición intrinseca de una nueva gobernan- 
za de la ciencia y la tecnologia, habida cuenta de que la participación pública puede ser comprendida a partir de su doble manifestación: por un lado, un tipo de participación espontánea o informal; por otro lado, un tipo de participación formal que responde a una necesidad de cambio y llama a la modificación de las técnicas, mecanismos y formas de organización utilizadas por la administración para generar respuestas más acordes con las necesidades ciudadanas.

La denominación de gobernanza de la ciencia y la tecnología incluye una gobernanza social basada no solamente en el aumento de la participación pública y su conocimiento potencial sino también en la aplicación de estrategias para su obtención y puesta en práctica. No se trata tanto de proponer el aporte que el público es capaz de ofrecer como un esquema obligado de los modos de hacer planes científicotécnicos, sino de justificar en parte cómo la ciudadanía puede ayudar a fortalecer la generación de conocimiento útil para sus propias prácticas y necesidades. El caso práctico de estudio realizado en la Comunidad de Madrid permitió hacer una revisión histórica de cómo se han tomado las decisiones sobre esta materia. Se constató que la participación pública en la elaboración de los planes autonómicos se ha venido ampliando, tanto en términos de sectores de interés local como en términos jerárquicos y funcionales de los participantes.

\section{Racionalidad de la participación ciudadana en el diseño de políticas científicas}

Defendemos la convicción de que para formular politicas de ciencia y tecnología es preciso establecer una relación más fuerte y estrecha entre los, que hacen estas políticas y la sociedad civil (Sanz y Santesmases, 1996; Banthien et. al, 2003). Esta relación fortalecida proporciona politicas más democráticas y legítimas (Petersen, 1984; López Cerezo et al, 1998), y responde a un propósito teórico que apunta al establecimiento de nuevos estándares de aceptación para la ciencia y la tecnología, a partir de la comprensión de cómo, por y para quién se crea el conocimiento (Shapin, 2000; Chopyak y Levesque, 2002; Elam y Bertilson, 2003). La participación ciudadana también implica un compromiso ético, en tanto permite la racionalización de la distribución del poder, de los riesgos, de los beneficios y las responsabilidades (Harvey, 1998; Webler y Tuler, 2002; Kasemir et al, 2003). En definitiva, constituye un cambio social que sustituye el tradicional modelo de relaciones de apoyo económico y confianza por parte de la sociedad y la satisfacción de demandas por parte de la ciencia (Chopyak y Levesque, 2002). El involucramiento del público en las decisiones politicas parece tan necesario que empieza a ser clara la exigencia de un mejor entendimiento conceptual y teórico de la participación pública (Webler y Tuler, 2000).

López Cerezo et al. (1998) señalan la existencia de varios factores relacionados con el aumento de la sensibilidad social sobre los asuntos de ciencia y tecnología:

1. Nueva imagen de la ciencia y de la tecnología. La imagen benefactora que habia proyectado la ciencia, el carácter idealizado de la comunidad científica y la relación de dar y recibir entre científicos y sociedad civil ha cambiado en las últimas décadas.

2. Nuevo papel de los expertos. El papel en ocasiones tecnocrático de los expertos al servicio de las administraciones públicas termina deteriorando los valores democráticos y hace necesario un nuevo rol para los científicos bajo el que no sean simplemente utilizados por el poder político para legitimar decisiones que justifican otros motivos.

3. Nuevo concepto de participación sociopolitica. El ciudadano es cada vez más consciente de que su participación no se agota en la elección periódica de representantes políticos sino que intenta profundizar en el sistema democrático a través de la presión y manifestación ante ciertas decisiones sobre la gestión y evaluación del progreso tecnológico.

4. Nueva imagen del hombre como miembro del medio biosocial. Los impactos del cambio científico-técnico son observados y percibidos por los ciudadanos de manera cada vez más directa, por tanto se impone un creciente sentimiento de responsabilidad con respecto a las decisiones que produzcan efectos cercanos al sujeto.

Por otra parte, la Unión Europea evalúa las posibilidades de éxito de la participación de la sociedad civil basándose en la idea de que la pluralidad mediatiza el fortalecimiento de la responsabilidad y transparencia de todos, la calidad y la legitimación de los procesos de toma de decisiones. Sin embargo, es cierto que el proceso participativo en sí no garantiza siempre la repartición adecuada del poder o la justicia social y aunque defender los derechos de los ciudadanos o garantizar la justicia social forman parte de los objetivos de la democracia, vivir en este sistema de gobierno no significa que contribuya consecuentemente a ellos (Todt, 2003). 


\section{El camino hacia la regionalización de la ciencia y la tecnología en Madrid}

El devenir histórico y la consolidación de las políticas científicas y tecnológicas en España responde a acciones particulares y situaciones contextuales internas y externas que fueron las más influyentes y explican el protagonismo de determinados actores, sus intereses y las orientaciones que dieron forma al sistema científico-técnico.

Los antecedentes sobre la "política para la ciencian en España a principios del siglo XX se identifican con los esfuerzos realizados por la Junta para la Ampliación de Estudios (JAE), de perfil homólogo a los Consejos británicos (Sánchez Ron, 1989; Muñoz, 2001). Pero el verdadero impulso de la política científica y tecnológica en España se genera después de la Guerra Civil y puede ser explicado por etapas, denominadas según el proceso histórico que se vivia en el Estado: período autárquico (1939-1950), período de desarrollismo (19501970) y el periodo de transición democrática (1980-1997) (Sanz Menéndez, 1997). A finales de los 70 los cambios en la esfera constitucional generan el Estado de las autonomias.

En el caso de la Comunidad de Madrid, no fue sino hasta 1990 cuando se lanza el I Plan Regional de Investigación de la Comunidad de Madrid (I PRICM) ${ }^{2}$, que fue tan tardío al encontrarse en Madrid la mayor parte de los institutos de investigación, el mayor número de integrantes de la comunidad científica del pais y algunos grupos representativos del sector empresarial que habian formado sistemáticamente parte de las propuestas e iniciativas expuestas en el Plan Nacional, por lo que desde un principio no hubo una preocupación prioritaria por realizar esfuerzos regionales en esta materia.

El primer plan de la Comunidad de Madrid nace por un lado como concienciación de que la investigación y el desarrollo tienen una vertiente regional ineludible, por lo que se pone en práctica una visión flexible y realista que complementaria las acciones previstas en el Plan Nacional y en los programas europeos en la materia. Se cumple asi con el Estatuto de Autonomía de la Comunidad de Madrid, que le otorga competencias en materia de fomento de la investigación (art. 26.15). Se trató de combinar el binomio entre la investigación científica y el desarrollo tecnológico para generar mejores condiciones de vida regional.

A finales de 1993, la preparación del II Plan Regional de Investigación de la Comunidad de Madrid (II PRICM) reco- piló, por un lado, las diferentes particularidades de la región; y por otro lado, se tomaron en cuenta las recomendaciones del Libro Blanco de la Política Cientifica en la Comunidad de Madrid. La combinación de ambas visiones resultó en la necesidad de promover el avance industrial, puesto que los mecanismos implementados fueron ampliamente orientados a aumentar los incentivos para la innovación tecnológica y la modernización de los procesos productivos, de tal modo que se lograra una mejor conexión entre investigación tecnológica y actividades empresariales.

El III Plan Regional de Investigación Científica e Innovación Tecnológica (III PRICIT) fue diseñado en principio para el cuatrienio 2000-2003. Los periodos para los que generalmente venían siendo elaborados eran de 4 años, pero se prorrogaron tanto el II como el III PRICIT. La extensión del segundo se vio motivada por el Decreto de la Ley de Investigación Científica, mientras que en el caso del III Plan las razones estuvieron relacionadas con cambios en la esfera politica nacional que complicaron las acciones de planificación en su momento y el cumplimiento cuatrienal. En cualquiera de los casos, el Consejo Científico de la Comunidad optó por mantener estos dos planes por un periodo de cinco años.

Las orientaciones de la Unión Europea, los resultados del proyecto ERICIT (Estrategia Regional de Investigación Científica e Innovación Tecnológica) ${ }^{3}$, la evaluación de los resultados del II Plan Regional y el producto de año y medio de reuniones, discusiones y reflexiones entre la administración regional con investigadores, centros públicos de investigación y empresas son las bases sobre las que se sostiene el III Plan Regional. Hay que añadir a esto la aprobación de la Ley de Fomento de la Investigación Científica Tecnológica en abril de 1999, de cuyos resultados se generaron un grupo de directrices que también ayudarian a orientar el Plan. Esta ley incluia la decisión de fomentar la cultura científica en la Comunidad de Madrid, contribuyendo a la toma de conciencia de que el ciudadano debía implicarse en temas que cada dia le afectan más directamente:

"La ciencia y la tecnologia han adquirido en los últimos tiempos una dimensión que supera el entorno público y universitario en el que tradicionalmente se han venido desarrollando. Los medios de comunicación... ...y las revistas de divulgación científico-tecnológicas han despertado el interés de los ciudadanos, especialmente el de los jóvenes. $Y$ es que la 
investigación ha dejado de ser una actividad de laboratorio, aislada de la realidad social, para entrar de lleno en la vida cotidiana. El ciudadano ha tomado conciencia de la importancia que la investigación y la tecnología tienen para el desarrollo futuro, como elementos de transformación de la sociedad» (III PRICIT, 2000: 9).

La propuesta de este Plan se interesa por conseguir la participación, el intercambio y la colaboración de todos los agentes del sistema de investigación y desarrollo, entre los que se cuenta el propio ciudadano. Se crea la línea de Cultura y Participación ciudadana cuyo objetivo fundamental es uabrir la experiencia científica a la mirada y a la reflexión del ciudadano", intentando un mayor acercamiento entre la ciencia y la sociedad a través de un proceso de divulgación, incorporación a la participación y posterior reflexión. La "Feria Madrid por la Ciencian y la "Semana de la Ciencia» han sido los mecanismos de acción utilizados para esta área ${ }^{4}$. Se reconoce que la participación ciudadana es limitada en las acciones científico-tecnológicas, pero no deja de valorarse la posibilidad de definir estos asuntos colectivamente entre los científicos y la sociedad. El ciudadano pasa a ser parte constitutiva en la realización del Plan Regional, pero es considerado como un actor al que hay que dirigir acciones de formación o de familiarización con la ciencia más que de participación directa en la identificación de necesidades y en el diseño del plan mismo. Por tanto, la inclusión del ciudadano en la toma de decisiones formales sobre ciencia y tecnología es de escasa tradición en esta región por lo que quizás esto explica, en parte, su ausencia en el ámbito de la organización y planificación.

La revisión de los planes da muestras de que, con el transcurir del tiempo, su diseño ha ido incorporando nuevos participantes y nuevos métodos para la determinación de las demandas y la definición de las estrategias de acción. Se han identificado actores para que cumplieran funciones como gestores, demandantes y ofertantes, se han planteado objetivos en relación con las diversas áreas prioritarias, para cada una de las cuales se han diseñado programas estratégicos con sus respectivos mecanismos de acción y financiación. En la presentación de este trabajo estos temas no han sido ampliamente considerados por cuestiones de espacio y porque lo que nos interesa destacar son los resultados de la investigación empírica realizada acerca del último Plan Regional de Investigación Científica e Innovación Tecnológica (IV PRICIT), en la que se analizan los aspectos relacionados con la ampliación de actores y sus percepciones acerca de los nuevos modelos de gobernanza de la ciencia y la tecnología.

\section{Ampliando los horizontes en el IV PRICIT de Madrid}

EI IV PRICIT, diseñado a lo largo de 2004, permitió el involucramiento de la comunidad científica y la comunidad empresarial regional, confiados en que «la territorialización o localización de las políticas de ciencia y tecnologia se ha manifestado más que como una respuesta a la mundialización, como un proceso paralelo y convergente a los vertiginosos cambios económicos y sociales que la han acompañadon (IV PRICIT, 2005: 31).

Para lograr el objetivo fundamental de crear un sistema de ciencia y tecnología apropiado para la Comunidad de Madrid, la Administración Regional hizo un llamamiento a la comunidad científica a través de la universidades y centros de investigación, asi como también a ciertas empresas: se les pidió que eligieran cada una de ellas a sus expertos para participar en la elaboración del nuevo Plan. Una empresa privada se encargó de la organización de las mesas de trabajo y exposición de las áreas claves previamente diseñadas a partir de informes de prospectiva tecnológica, de las tendencias de la Unión Europea y nacional, y de los resultados de evaluaciones del Plan anterior. El proceso de trabajo se desarrolló a lo largo de tres encuentros en los que se les pidió a los participantes que expusieran sus opiniones, establecieran las prioridades y generaran sugerencias. Durante el proceso se fueron afinando los resultados y las sugerencias a través de la red. De tres reuniones de trabajo se obtuvieron veinte áreas prioritarias ${ }^{5}$. Posteriormente, los informes generados por cada grupo pasaron a manos de la Secretaria del Plan para la evaluación de los mismos y la definición de los mecanismos de acción.

En el nuevo Plan se plantea la continuidad de dotación de recursos y capacidades del sistema regional de ciencia y tecnología, la integración, cooperación, utilización y consolidación de las potencialidades del medio en su conjunto, tanto dentro como fuera de la región, así como también en el ámbito internacional. Lo relativamente nuevo y no menos importante en el interés de sus objetivos es la implicación de la sociedad madrileña en general en la empresa científica, así como el mayor empeño en la divulgación de la política regional de ciencia y tecnología. El monto inicial del Plan es de 225 millones de Euros repartidos de la siguiente manera: 
TABLA 1. Distribución inicial de la financiación del IV PRICIT según actuación y dedicación

\begin{tabular}{|l|c|}
\hline Grupos de investigación & $25 \%$ \\
\hline Dotación de infraestructura de interés regional & $25 \%$ \\
\hline Creación de capital humano & $30 \%$ \\
\hline Fomento de la cooperación y de la I+D empresarial & $10 \%$ \\
\hline Ciencia y Sociedad & $5 \%$ \\
\hline Gestión, seguimiento y valuación & $3 \%$ \\
\hline Cooperación interregional & $1 \%$ \\
\hline Propuestas institucionales & $1 \%$ \\
\hline
\end{tabular}

\section{Descripción metodológica del estudio}

El estudio empírico realizado ha consistido en la selección de 9 de las 20 áreas incluidas en el diseño del Plan, a saber: Energía, Materiales y nanotecnología, Tecnologías de la información y de las comunicaciones, Recursos naturales y tecnologias medioambientales, Repercusión social de la investigación, Evaluación de la gestión e instrumentos del Plan, Socioeconomia, humanidades y derecho, Ciencias de la salud y Espacios europeos de investigación. Se escogieron estas áreas por considerar que son aquellas con mayor probabilidad de abrirse al debate público y porque de alguna forma son las que pueden generar efectos directos en la vida diaria de la ciudadanía. Posteriormente, se seleccionaron algunos participantes que formaron parte de estos grupos de trabajo priorizando la diversidad entre los entrevistados: científicos teóricos, experimentales, tecnólogos y gestores, entre otros, por lo que la formación de los entrevistados va desde graduados en ingenierias y ciencias básicas hasta ciencias sociales y humanas, lo que ha dado una visión multidisciplinar a la temática en estudio. Se diseño una entrevista semiestructurada, destacando básicamente cuatro ejes temáticos: i) Gobernanza cientifico-tecnológica, ii) Participación pública en el diseño de politicas científico-tecnológicas; iii) Participación pública en decisiones científico-tecnológicas; iv) Participación ciudadana en el Plan de ciencia y tecnologia de la Comunidad de Madrid. El total de los participantes en los grupos de trabajo suman 267 actores y la muestra entrevistada y analizada representa un $6,7 \%$ de ese total, siendo la participación femenina de un $25 \%$ siguiendo la tendencia dada en el universo.

\section{Actual sistema científico-tecnológico de la Comunidad de Madrid}

El diseño de los planes de ciencia y tecnologia en cualquiera de sus niveles y con participación de la sociedad civil supone una intervención organizada, gestionada y planificada principalmente desde la administración. Los planes son productos públicos, que integran una pluralidad de niveles de participación, de agentes de necesidades y prioridades y mecanismos de acción. Como producción pública su diseño debe reunir en sus procesos de participación una triple composición: consensuada, es decir, que involucre la canalización y racionalización acotada de todos los puntos de vista afectados, material, traducida en las prácticas de los actores; y jurídica, respaldada por una legislación pertinente para alcanzar los objetivos propuestos.

El diseño del IV PRICIT se caracterizó por tener el funcionamiento de un sistema abierto y participativo, desde el proceso de convocatoria hasta la resolución de prioridades, a través de un consenso deliberativo que respondió a la voluntad de incorporar nuevos actores. Hay que añadir que se insiste en una mayor apertura o consenso en la etapa de toma de decisiones finales (realizada por la administración) para hacer del Plan una tarea completamente abierta a la sociedad. En la elaboración del Plan interaccionaron expertos, administradores, técnicos, gestores y empresarios: «...en ese sentido fue horizontal y democrático. La gente se siente muy bien tratada donde están todos de igual a igual, en este caso todos eran vistos como pares, es participación ciudadana aunque fuese cualificada, escuchar un poco sus opiniones; todo el mundo participa para escuchar, resumir y tomar nota de lo que alli se decian (E8) ${ }^{6}$.

Por otra parte, los entrevistados coinciden en que la demanda social es un criterio clave a considerar en la elaboración de los planes, lo que aumenta las probabilidades de que los ciudadanos puedan participar en la identificación de necesidades o prioridades. "La opinión ciudadana siempre es fundamental, pero sólo se ve cuando está directamente afectada. La participación ciudadana es fundamental, asi como se pide opinión a los expertos, es un deber, el dinero es público, 
de todos los ciudadanos, por lo que hay que tenerlo en cuenta, a los ciudadanos al menos para que nos digan en qué quieren que se investigue más» (E10).

\section{La participación pública en planes de ciencia y tecnología de la comunidad de Madrid. ¿Ejercicio de voluntad o convocatoria?}

En el marco de los planes regionales de investigación científica e innovación tecnológica cobra cada vez más fuerza la idea de que resulta pertinente determinar los modelos de toma de decisiones que caracterizan el diseño de éstos y por qué, en qué tipo de conocimiento experto se basan, qué medios y criterios utilizan para la formulación de sus objetivos, qué actores participan (y quiénes no) durante el proceso (Olazaran et al, 2004) y la pertinencia de incluir a expertos sociales en la etapa de intercambio y toma de decisiones.

La participación del público puede percibirse a través de diferentes tipos o procesos de participación, según sea el caso en debate. Por un lado, está la participación informal o espontánea dada cuando la ciudadanía se expresa sin ser convocada y obedece a las acciones abiertas de grupos sociales que se organizan para solicitar, refutar o hacer valer su opinión. Por otro lado, está la participación formal relacionada con el cumplimiento de una elaboración sistemática de actividades en las que existe un grupo interesado en reunir a diversos representantes 0 agentes sociales con la finalidad de intercambiar puntos de vista que permitan generar un trabajo, documento o tecnología con proyección de futuro.

La participación espontánea por lo general responde a situaciones de controversias o conflictos. En la participación formal o sistemática se incluyen todos aquellos estilos que siguen un procedimiento consecuente para recoger e intercambiar opiniones. La finalidad principal es establecer un diálogo entre los afectados o interesados en un tema relacionado con la ciencia y la tecnologia 7 . El denominador común de estos procesos de participación es que combinan conocimientos diversos, otorgando un papel relevante a la sociedad civil, siguiendo procedimientos formales y tomando decisiones tras un proceso negociador entre las partes. Se privilegia el diálogo 8 como factor clave para acercarse a resultados legitimos.

En el estudio realizado se observa que los planes de ciencia y tecnología de la Comunidad de Madrid se caracterizan por tener una participación pública determinada por procesos formales de participación, se trata de una participación diversa en cuanto a sectores sociales (económicos, cientifi$\cos$, académicos, industriales, políticos, tecnológicos), aunque no contempla la intervención del ciudadano no experto. La participación dada en el último plan corresponde a un proceso de convocatoria elaborado de manera sistemática por la administración. Algunos de los participantes señalan que el proceso debería ser abierto de principio a fin, es decir, "las prioridades deben tomarse por deliberación de los agentes que participan con intereses diferenciados; las comisiones de diseño tienen que llegar a consenso y se llega parcelando las prioridades. Deben ser unas prioridades plurales en las que, en mayor o menor grado, estén representadas las prioridades de los agentes sociales. Un gobierno no puede hacer el papel de gobierno y mando, si es democrático no puede llevar sus propias líneas prioritarias, sino que los planes hay que hacerlos previa deliberación buscando los agentes relevantes y consenso. En caso que no haya consenso se puede ir a evaluaciones externas. El gobierno debe funcionar como un coordinador y armonizador de los intereses que surjan» (E6).

En el actual ámbito regional, la demanda social no parece estar articulada a los planes, pero buena parte de los entrevistados coinciden en la conveniencia de aumentar la diversidad de la base definitoria de las políticas científico-tecnológicas, considerando que "toda aportación es enriquecedora en sí misma y cuanto más diversa sea la aportación más iniciativas hay" (E14). Además, "la ciencia es más cara. Esto se vuelve cada vez más competitivo internacionalmente hablando. Las politicas también generan resistencia y crean más conflicto dentro y fuera de la comunidad cientifica, entonces, ampliar la base de apoyo a esas políticas parece imprescindiblen (E11).

La extensión o apertura de los procesos de participación también se debe a una necesidad latente en la sociedad como parte de su propio carácter evolutivo: "La propia evolución de la ciencia y la tecnología lo exigen, sobre todo por sus impactos sociales, económicos, ecológicos, militares, etc. A medida que se va hacia la sociedad de la información y el conocimiento, el conocimiento científico-tecnológico pasa a ser el nuevo motor de la nueva modalidad de sociedad; el conocimiento es el motor de riqueza, de poder y de innovación. Esto afecta a la sociedad en su conjunto y, por lo tanto, la ciencia actual ya no es patrimonio de los científicos, sino que la tecnociencia (ciencia contemporánea), en la medida 
que incide y tiene enormes consecuencias sobre muchos ámbitos del mundo, entonces los programas de la ciencia y desarrollo que se va a hacer tiene que involucrar a esa gente. Ya no se puede pensar en el monopolio del conocimiento científico, los científicos tienen que diseñar sus propios objetivos pero con otros agentes" (E6).

Aquellos pocos entrevistados que mostraron cierto escepticismo por el aumento de la pluralidad de agentes participantes en la elaboración de las politicas en ciencia y tecnología lo hacen enfatizando cierta preocupación en el bajo nivel de alfabetización científica de la ciudadanía. Este punto merece un tratamiento aparte que retomaremos más adelante.

El principal obstáculo identificado por los entrevistados para la implicación de los ciudadanos en los procesos de planificación científico-tecnológica es la falta de mecanismos de participación. Los entrevistados han expuesto algunas propuestas que coinciden con los procesos de participación formal mencionados anteriormente:

TABLA 2. Propuestas metodológicas para promover la participación pública en políticas científico-tecnológicas

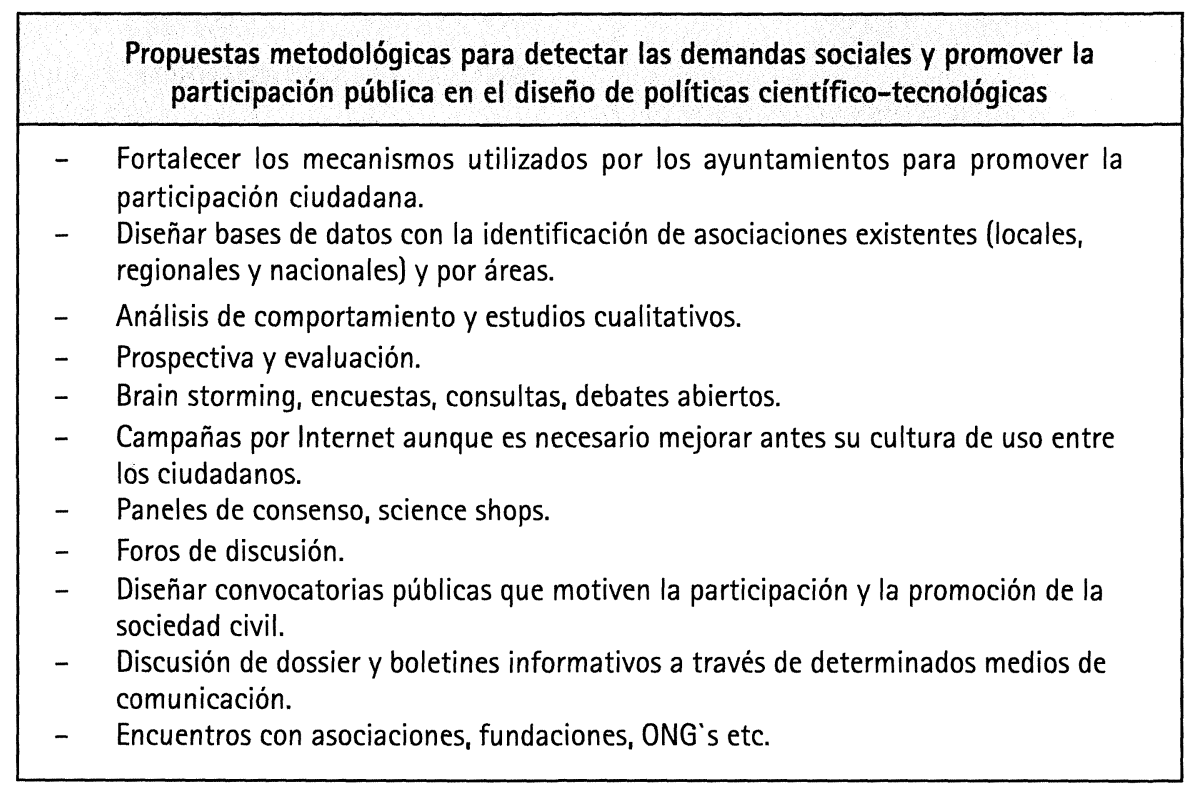

\section{Política científica y tecnológica. Los inicios de la nueva gobernanza y la gobernanza social}

La política cientifica "designa las acciones intencionales desarrolladas por los gobiernos, o en sentido más amplio por el Estado, con el objetivo de apoyar, promover o influenciar el desarrollo de la ciencia y la tecnología. La noción de intencionalidad apunta a que las acciones o programas emprendidos por el poder político a través de diversos mecanismos deben tener como propósito explícito (esperando y deseando) influenciar, promover o apoyar el desarrollo de la ciencia y la tecnología, del conocimiento en general o de sus aplicaciones especificas» (Menéndez, 1997: 17).
La idea general sobre la función de la politica de ciencia y tecnología está influenciada por la existencia de un conjunto de técnicas inmateriales ejecutadas por un grupo de actores especificos y en un momento determinado (gestores, demandantes y receptores), para la realización de actividades que forman parte de un sistema interdependiente, persiguiendo el beneficio de las partes involucradas y de la sociedad en general. Cabe destacar que, en el estudio realizado, se observa una gran inclinación, por parte de los científicos académicos, hacia la idea de una distribución ampliada de los recursos entre posibles implicados, sectores y acciones, mientras que los tecnólogos toman más en consideración el fortalecimiento competitivo del sistema. Probablemente esto se debe a que las innovaciones de los tecnóARBOR CLXXXI 715 SEPTIEMBRE-OCTUBRE [2005] 363-375 ISSN: 0210-1963 
logos son innovaciones inmediatas para la industria o la empresa mientras que los científicos académicos y los gestores tienen una visión más amplia de los miembros que integran el sistema y en los que se produce sus efectos directamente.

Para la Unión Europea, la nueva gobernanza se presenta como un desafío ante la necesidad de una acción urgente para adaptar el gobierno a los asuntos existentes y ante la necesidad de ampliar el debate sobre el bienestar de Europa. El desafío para lograr los cambios constituye una acción de esfuerzo conjunto de las instituciones administrativas (gobierno central, regional, local) y la sociedad civil para dirigir, desde los actuales estados miembros, el futuro de la Unión Europea (Comisión Europea, 2001).

En gran medida, la gobernanza europea se traduce en "reglas, métodos, procesos y comportamientos que afectan la forma en la cual los poderes se ejercen a nivel europeo, considerando particularmente como principios la apertura, la participación, la responsabilidad, la eficacia y la coherencia de las propuestas" (Libro Blanco de la Gobernanza Europea, 2001). Estos principios son significativos por si mismos pero no pueden ser alcanzados a través de acciones separadas. Por esto, las políticas no pueden ser efectivas a menos que se preparen de una forma más inclusiva.

La propuesta de la Unión Europea es una visión que apuesta por una reforma de la política científica y tecnológica basada en una democracia reflexiva y priorizando la legitimidad social de las actividades, pero sigue siendo un asunto en plena elaboración.

En el estudio empírico realizado en la Comunidad de Madrid, los entrevistados que participaron en la elaboración de planes relacionan también el término "gobernanza" con una nueva forma de estructuración y organización de la agenda política, que incluye una renovación de la práctica democrática en la que se desenvuelven los procesos de toma de decisiones en la esfera pública, poniendo en práctica algunos de los esquemas de organización participativa ya mencionados.

En el caso regional, la gobernanza de la ciencia y la tecnología "implica la forma como se gobiernan estos asuntos en diferentes niveles administrativos" (E16). "Tiene que ver con el diseño de los sistemas de opinión y con los sistemas ejecutivos» (E4). Siguiendo la tendencia europea, "es una nueva visión de cómo debe ser el gobierno, de cómo tiene que ser la acción política en determinados ámbitos, no sólo de la ciencia y la tecnología. Lo que se pretende con esta nueva idea es fomentar la participación de todos los agentes involucrados en una politica de ciencia y tecnologia" (E10).

Explican también la gobernanza como un proceso elaborado de "toma de decisiones con la mayor información que se disponga no sólo científica, ni económica (...) además, la sociedad quiere saber qué se está haciendo con su dinero, en qué se está invirtiendo" (E7). "Cómo manejar una determinada tecnología que no sólo incluye la promoción sino también la regulación y demás implicaciones sociales; es un proceso que involucra muchos actores y genera una trayectoria sostenida de representantes" (E12).

"Es algo que está de moda, como necesidad, en la medida en que la sociedad se hace cada vez más compleja, las instituciones que deben intervenir son cada vez más, el número de actores es cada vez mayor, los ámbitos son cada vez diferentes, considerando que todo además está entrelazado, que casi no hay fronteras. La idea que está detrás de la gobernanza es importante desde la perspectiva de la democratización de la ciencia y la tecnología” (E5).

La inclusión de la sociedad civil en la gobernanza puede ser considerada como una renovación de la acción pública basada en dos aspectos fundamentales: uno es el que tiene que ver con una reconstrucción de la democracia participativa y la evolución de las formas de administración política en la que se da una vinculación más abierta y activa de los actores implicados en la toma decisiones científico-tecnológicas. El otro aspecto está dado por la incorporación del conocimiento de la ciudadanía a través de la convocatoria dirigida a algunos representantes pertenecientes a colectivos o asociaciones civiles altamente implicados o afectados en los asuntos sociales tratados en las politicas.

La gobernanza científico-tecnológica puede ser explicitada también por su soporte metodológico, que integra y gestiona una diversidad de instituciones pertenecientes a diferentes grupos o campos cientificos (multisectores) que, trabajando bajo un esquema multifuncional (es decir, los actores, además de trabajar en su área, comparten y participan en otras actividades relacionadas con su sector de trabajo pero en un espacio de planificación conjunto con otros ámbitos de la esfera pública), cumplen diversas funciones y 
ejercen diferentes roles (multijerarquias). La finalidad de esta vinculación es generar un mayor conocimiento, introducir nuevas tecnologías, diseñar determinadas políticas y aumentar la calidad de vida del entorno. Igualmente el resultado es producto de la aplicación de modelos y dispositivos (multimodelos; multimecanismos) según el terreno de debate y objetivos planteados. La utilización de multivariables se convierte en el rasgo más destacado de la nueva gobernanza de la ciencia y la tecnología, ampliando así la legitimación social y epistémica de esta propuesta y sus contenidos.

La nueva propuesta de gobernanza puede ser entendida como una gobernanza social al integrar como parte de su núcleo central la sociedad civil, lo que incluye, a su vez, implicaciones políticas en tanto se le considere como parte agregada con voz para proponer líneas de acción y capacidad para formar parte del grupo de decisores últimos; también contempla implicaciones epistémicas en tanto se necesita poner en marcha un conjunto de normas, modelos o métodos, técnicas, etc, que vigilen la veracidad, sistematicidad y cumplimiento justo de una participación equilibrada.

\section{Hacia un análisis pentagonal de la gobernanza científica y tecnológica}

El último plan autonómico de la Comunidad de Madrid ofrece indicios de una reorientación en el sistema de gestión, dada la apertura de la participación a diversos expertos y las potencialidades que generaria un posible acceso de expertos sociales en algunas áreas básicas. En la diversidad que representa la nueva gobernanza, uno de los ejes más destacados es, precisamente, la inclusión o participación de diferentes actores. La relevancia de los actores y sus opiniones es destacada aqui porque a partir de ellos y del rol que desempeñan se desencadena el resto de las multivariables incluidas en la nueva gobernanza de la ciencia y la tecnología. La propuesta que planteamos, sustentada sobre las percepciones y reflexiones de los participantes entrevistados, se proyecta en la presentación de una base pentagonal, compuesta por esos actores principales para reorientar el diálogo desde la administración. Desde esta configuración, la elaboración de planes regionales sobre ciencia y tecnología se caracterizaria por ser producto de una red de relaciones, mediante la cual se construye conocimiento y se plantean propuestas de acción, a partir de metodologías basadas en programas estratégicos de participación.
Uno de los ángulos que forma parte de esa base pentagonal para el diseño de política cientifica y tecnológica es el desempeñado por el científico o experto, el tipo de actor que, tradicionalmente ha estado más cerca de la administración en la relación Gobierno/Investigación. Los científicos son quienes ejercen el rol de especialistas en las áreas científico-tecnológicas tratadas; ya que se les reconoce capacitación para señalar o proponer las necesidades en su campo de estudio o sugerencias del sistema en general. A su vez, su preparación técnica les permite detectar y prevenir riesgos o posibles desventajas. El científico es visto como un actor con visión privilegiada dentro y fuera sistema administración/investigación. Además, actúa como el principal ente de comunicación y portador de información, que debe ser discutida con otros expertos y el resto de actores para posteriormente ser procesada por la administración y finalmente difundirla a la sociedad.

El Gestor es un actor cuyo rol básicamente es el de gestionar los recursos de la investigación. Es quien pone en práctica metodologias para reunir al resto de actores involucrados, procesa y genera las propuestas de acción, gestiona los mecanismos para desarrollar las politicas (convocatorias, convenios, contratos, etc), y quien vela por el cumplimiento de las reglas del juego a lo largo tanto del proceso de elaboración del plan como de su ejecución y evaluación.

El rol representado por el Político está muy asociado al trabajo de gestión que desempeña la administración, pero el papel de los políticos está más ligado al diagnóstico de la situación y de cuáles deberian ser las directrices de la politica regional de la Comunidad; han de tomar en cuenta cómo se han hecho las cosas, cómo se tienen que hacer e identificar conjuntamente con los gestores de la administración los éxitos y fracasos de las acciones. El político forma parte del grupo que avala los presupuestos para poner en marcha los mecanismos de acción y que apoya o no las estrategias que desarrolla la administración como parte de las acciones científico-tecnológicas, "tiene la obligación de repartir los recursos públicos, hacer una distribución equitativa, razonable y eficaz de esos recursos» (E2).

Las capacidades de desarrollo de los sistemas de I+D de las empresas constituyen otra de las bases esenciales en el análisis propuesto, ya que en gran medida tienen un peso relevante en la orientación de las políticas de investigación y desarrollo nacional y regional. Según el Informe COTEC 2004, la Comunidad de Madrid para el año 2002 registraba

ARBOR CLXXXI 715 SEPTIEMBRE-OCTUBRE (2005) 363-375 ISSN: 0210-1963 
en gastos de I+D un total de 2.277,8 millones de euros representando un $58,1 \%$ el sector empresarial y un $41,9 \%$ el sector público. Para la administración misma y el resto de los agentes ya es un hábito asentado contar con la participación de representantes empresariales para la elaboración de los planes regionales. Los sistemas de I+D de las empresas trabajan en red con otros actores del sistema cumpliendo funciones de investigación, formación y satisfacción de demandas para cuyas acciones se justifica parte de la elaboración del Plan.

El último, pero no menos importante ángulo de la base pentagonal, corresponde a la Ciudadanía. Puede presentarse en dos formas, como receptor o usuario de las innovaciones, y como parte de los demandantes que expresan sus expectativas y prioridades en el sistema. Ciertamente, el público general tiene una importante carencia de canales de negociación, lo que sumado a los cambios que ha registrado la institución científica, la generación de controversias y la implicación directa de los ciudadanos en los efectos que produce el avance tecnocientífico, ha dado lugar a la organización de la sociedad civil que canaliza sus intereses a través de ONGs, asociaciones, fundaciones o grupos de consumidores. Estas organizaciones funcionan como agentes intermediarios a través de los que la sociedad civil puede expresar sus demandas y conocimientos, proporcionando así un nuevo empoderamiento de los ciudadanos en esta esfera público-administrativa (Funtowicz et al, 2002).

A pesar de que en la Comunidad de Madrid todavía no son claros los mecanismos más apropiados para incorporar a la sociedad civil ${ }^{9}$ en la toma de decisiones del sistema científico, su incorporación es vista como un ingrediente de equilibrio al sistema. Sin duda la experiencia muestra que "su grado de incorporación hasta el momento es pequeño pero probablemente en las décadas venideras no dejará de aumentar, entre otras cosas por las implicaciones sociales, éticas, etc, de la misma política científica de hoy día; cualquier decisión que afecte a la ciencia afecta directamente a la sociedad y en consecuencia quiere involucrarse y participar más en las decisiones que se van a tomar» (E5). La idea de participación ciudadana en la que se inscribe la propuesta pentagonal se basa en la identificación y propuesta de expertos sociales que cumplan la función de representantes o delegados proveniente de las diversas organizaciones e instituciones cuyos intereses les identifican como una ciudadanía cualificada. Puesto que "es dificil hacer que la gente participe de manera directa, es necesario generar agentes intermedios, la interlocución no puede ser directa entre científico y ciudadano, es necesario generar cultura cientifica (instituciones donde se encuentran interlocutores, gente más sensibilizada)» (E1). La inclusión de expertos sociales contribuiría a la mejora del conocimiento eficaz necesario para el diseño y puesta en funcionamiento de los mecanismos de acción propuestos en el Plan. No se trata de conocimiento técnico como el que aporta cualquiera de los otros especialistas del sistema, ni siquiera el propio de los científicos sociales, sino que su aportación se basa más bien en el conocimiento práctico de las demandas concretas y de variables que pasan desapercibidas a los expertos tradicionales.

La perspectiva pentagonal de la gobernanza de la ciencia contiene la combinación de una nueva composición en la que puede evidenciarse la evolución organizacional del sistema científico-técnico y el alcance, aún más eficaz, de sus objetivos mediante la innovación en la producción del propio conocimiento.

Los resultados del estudio señalan así una alta tendencia a promover la participación ciudadana en la planificación del plan regional de ciencia y tecnología. Los mismos administradores entrevistados ven como tarea pendiente esta acción y se muestran receptivos a sugerencias para convertirla en una de sus metas en el diseño de futuros planes, pues: "Ios problemas sobre los que hay que tener criterio y tomar decisiones hace ya mucho tiempo escaparon del control de los expertos, de los laboratorios, de las aulas de las universidades y el caso es que ahora ya lo sabemos todos, lo saben los políticos, los expertos y hasta los mismos ciudadanos, entonces, todos quieren tener un papel dentro de esta toma de decisiones» (E11).

Hasta ahora la falta de participación de la sociedad civil en la programación de planes regionales responde básicamente a tres razones fundamentales: no hay tradición participativa en el área, existe un déficit de mecanismos puestos en práctica desde la administración para que ésta se lleve a cabo, y el nivel de cultura científica de la ciudadanía es considerado bajo: "Vivimos en una sociedad y en un sistema de ciencia y tecnología desvertebrado y no hemos sido capaces de generar instrumentos que posibiliten la interacción entre los ciudadanos, destinatarios últimos de los beneficios que deben producir los investigadores» (E1). "El nivel cultural y científico de este país es muy bajo. Además no hay tradición de participación ciudadana en el área, esto lleva décadas 
para que pueda estar implantado y para que exista cierta tradición. Si hay cierto interés por la ciencia, pero el interés sigue siendo bajisimon (E8).

La experiencia internacional también concluye que la participación del público en la resolución de problemas es determinante, por lo que se ha tratado de extender una creciente conciencia e integrar la consulta del público general y otros actores sociales como uno más de los requisitos planteados para financiar proyectos de investigación (Natenzon y Futowicz, 2003). De hecho, esto ha sido propuesto como tema a considerar en el diseño del próximo plan de la Comunidad de Madrid: "si a cada propuesta de investigación que solicite apoyo financiero se le exige, como parte de sus condiciones a cumplir, que incluya o esté relacionada con cualquier grupo, organización civil, ONG, u otro tipo de asociación que demuestre su interés o preocupación en el campo de investigación propuesto, entonces se dará más crédito a una propuesta que promueva tales relaciones" (E1). Se trata de promover la creación de redes abiertas de investigación y aprendizaje entre quienes producen buena parte del conocimiento y generan servicios o productos más estrechamente vinculados con las demandas sociales.

\section{Consideraciones finales}

Desde la segunda mitad del siglo XX la ciudadanía ha venido viviendo una nueva etapa de sensibilización social en relación con la ciencia y la tecnología, relacionada a menudo con la cara más amarga de los efectos de los avances científicos y desarrollos tecnológicos. La sociedad, además de ser testigo, recibir y apoyar el desarrollo cientifico, reclama ahora un rol como partícipe de la orientación científica y tecnológica. En este sentido, el nivel regional puede proporcionar una buena plataforma para esta implicación activa.

El diseño de los planes regionales de la Comunidad de Madrid se caracteriza por una clara evolución, mostrando los cambios en los modelos lineales de innovación y la transición hacia formas de gobernanza. La implicación de un mayor número de instituciones y la aplicación de principios, normas y estrategias de legitimación social son las claves de esta transformación.

Entre los participantes entrevistados existe una amplia aceptación de las potencialidades de interactuar con representantes de organizaciones sociales (expertos sociales), pues no se duda de la capacidad de estos actores para relacionarse con el resto de los representantes institucionales, dialogar y proponer los criterios de la orientación científico-tecnológica socialmente más aceptada. Aunque se indica que quizás no todas las áreas científico-técnicas pueden ser abiertas al debate público, existen algunas como la salud, el ambiente, la alimentación, el transporte, la energia, etc, que resulta esencial someter a negociación social.

La inclusión de la participación ciudadana se contempla como una evolución metodológica en la forma de hacer la política científica que enriqueceria los programas de acción. Para integrar este nuevo grupo se propone la selección de encargados de asociaciones, organizaciones $u$ ONG's a quienes se les denomina expertos, delegados 0 peritos sociales quienes son los que probablemente tienen mayor disposición y conocimiento para interactuar con el resto de los actores participantes. Otra de las materias pendientes en esta gestión es poner en marcha estrategias que permitan la interacción con esos expertos sociales, para lo que se proponen diversas metodologias de participación ciudadana.

Finalmente, la nueva gobernanza de la ciencia incluye un sistema multivariable dentro del cual sus actores claves deben embarcarse en un intercambio basado en el diálogo propiciado a partir de los mecanismos utilizados para producir una mayor participación e intercambio entre las redes, privilegiando el interés o la visión del receptor directo de los efectos de las decisiones tomadas. 


\section{NOTAS}

1 Este trabajo forma parte del proyecto "Sociedad civil y gobernanza de la ciencia y la tecnologia en Españan, realizado en el departamento CTS del Instituto de Filosofia, CSIC y financiado por la Fundación Española para la Ciencia y la Tecnología, FECYT. Agradezco a todos las personas entrevistadas su disponibilidad para la colaboración en este estudio; así como a Marta I. González Garcia las observaciones hechas para la preparación de este artículo.

2 Existen otras comunidades autónomas que ya desde el periodo preautonómico realizaron actividades relacionadas con la política científico-tecnológica. La Comisión Asesora de Investigación y Desarrollo Tecnológico (CAIDT) y el Comité Vasco de Tecnologia (CVT) en la Comunidad Autónoma del Pais Vasco (CAPV) confluyeron posteriormente en el Primer Plan de Desarrollo Industrial. Para más detalle véase Moso, 1999.

3 Es un proyecto que se encuentra dentro del programa RITTS (Regional Innovation and Transfer of Technology Strategy) promovido por la Dirección General XIII de la Comisión Europea.

4 A pesar de que la propia comunidad académica y científica no consideraba este ámbito como una de las líneas prioritarias dentro de un plan de investigación e innovación. Fue necesario un cambio gradual que subrayara el valor del conocimiento científico y tecnológico para la ciudadania, de manera que les capacite para entender los procesos de la ciencia y decidir sobre las políticas científicas. Uno de los impactos positivos de esta área de trabajo se desglosa en detalle en los resultados y evaluación de la Feria Madrid por la Ciencia en el trabajo de Almudena del Rosal (2004).
5 i) Energia, ii) Materiales y nanotecnologia, iii) Tecnologias agroalimentarias, iv) Tecnologias de la información y la comunicación, v) Ciencias de la Salud, vi) Recursos naturales y tecnologías medioambientales, vii) Productos y procesos químicos, viii) Socioeconomia, humanidades y derecho ix) Ciencias del espacio, física y matemáticas, $x$ ) Recursos humanos en $I+D+I$, xi) Apoyo a grupos de investigación, xii) Bienes de equipo, diseño y producción industrial, xiii) Infraestructura para la $I+D+I$, xiv) Repercusión económica de la investigación, xv) Repercusión social de la investigación, xvi) Espacio europeo de investigación y relaciones con lberoamérica, xvii) Evaluación de la gestión e instrumentos del Plan, xviii) Investigación en la red de hospitales, xix) Coordinación del Plan con las estrategias de las universidades, $x x$ ) Entorno empresarial e innovación.

6 Para preservar el anonimato de las personas entrevistadas, en aquellos casos en los que se hacen citas textuales de las mismas se identifican con la clave Ex, donde $x$ es un número asignado al azar a cada una de las entrevistas realizadas.

7 Se incluyen en este grupo las conocidas formas de participación tales como: audiencias públicas, conferencias de consenso, encuesta de opinión, gestión negociada, congreso de consenso, panel ciudadano, mediación, debate nacional, audiencia parlamentaria, oficina de evaluación de tecnologías, evaluación constructiva de tecnologias, comunidad de pares ampliada, agendas de ciencia y tecnologia, forum de ciencia, litigio, consumo diferencial, protesta pública, comité asesor de ciudadanos, análisis integrado, grupos de discusión, foro de debate, investigación de base comunitaria, boutique de la ciencia, comités de trabajo, referéndum, entre otras (Petersen, 1984; Chopyak y Levesque, 2002; López Cerezo y González García, 2002; Banthien et al, 2003; Kasemir et al, 2003).
8 Kasemir et al. (2003) presentan varias acepciones teóricas para el entendimiento del diálogo como procedimiento determinante que permita la participación equilibrada de los interesados. A saber: diálogo como negociación, diálogo como entendimiento, diálogo como dominación, diálogo como sentido común.

9 En el IV PRICIT la posibilidad de participación ciudadana se dio exclusivamente por medio de la página web de la comunidad, en la que se publicaban los informes que iban generándose y en la que se ofrecía la opción de participar, hacer sugerencias o peticiones. La participación en este sentido fue prácticamente nula y las razones a las que obedece este hecho es que probablemente el público no estaba al tanto de tal proceso o porque el medio de participación, por ahora, no resulta ser el más idóneo. 


\section{BIBLIOGRAFÍA}

Banthien, H, M. Jaspers \& A. Renner (2003): Governance of the European Research Area "The role of civil society». Comision Europea, IFOK Interim Report. $<$ http://europa.eu.int/comm/research/ science-society/>.

Canto, R. (2000): Políticas públicas. Más allá del pluralismo y la participación ciudadana, Gestión y Política Pública, 9/2, 231-256.

Comisión de las Comunidades Europeas (2001): European Governance. A white paper. Bruselas.

Comisión de las Comunidades Europeas (2002): Communication from the Commission on the collection and use of expertise by the Commission: Principles and Guidelines. Improving the knowledge base for better policies. Bruselas.

Cotec (2004): Informe 2004. Tecnología e Innovación en España. Madrid:

Fundación COTEC para la Innovación Tecnológica.

Chopyak, J. \& P. Levesque (2002): Public participation in science and technology decision making: trends for the future. Technology in Society, 24, 155-166.

Del Rosal, A. (2004): El reto de la Administración: Ia ciencia y los ciudadanos, en: Rubia, F. et al. (eds.): Percepción social de la ciencia. Madrid: Academia Europea de Ciencias y Artes (UNED).

Edler, J, Kuhlmann S. \& M. Behrens (2003): Changing gobernance and technology policy. The Europea Research Area. Massachusetts: Edward Elgar Publishing Limited.

Elam, M. \& M. Bertilsson (2003): Consuming engaging and confronting science. The emerging dimensions of scientific citizenship, European Journal of Social Theory, 6/2, 233-251.

Funtowicz, S. \& J. Ravetz (1993): Science for the pos-normal age, Futures, 25/7, 735-755.

Funtowicz, S. et al, (2000): Science and governance in the European Union: a contribution to the debate, Science and Public Policy, 27/5, 327-336.
Harvey, R. (1998): Modern Science: Institutionalization of Knowledge and Rationalization of Power, en: Toward a Democratic Science. Scientific Narration and Civic Communication. Yale University Press, 122- 152.

Kasemir et al., (2003): Public participation in sustainability science. A handbook. Cambridge: Cambridge University Press. Lebessis, N. \& J. Paterson (2000): Developing new modes of governance, Working paper - 2000. European Comission. Forward Studies Unit.

LFCGICT (2002): Ley 13/1986 de 14 de abril. Ley Fomento y Coordinación General de la Investigación Científica y Técnica. $<$ http://www.goico.net/legis/univ/ coordinainves.htm>

LFICIT (1998): Ley 5/1998 de 7 de mayo. Ley de Fomento de la Investigación Científica y la Innovación Tecnológica <htt://www.madrimasd.org/ informacion/estrategia/doc/ley51998.pdf>

López Cerezo, J. A. et al., (1998): Participación pública en política científica y tecnológica. problemas y perspectivas, Arbor, CLIX/627, 279-308.

López Cerezo, J. A. \& M. I. González (2002): Politicas del bosque. Madrid: Cambridge University Press/OEI.

Moso, M. (1999): Origen y evolución de las políticas cientificas y tecnológicas en la Comunidad Autónoma del País Vasco. San Sebastián: Servicio Editorial UPV/EHU.

Muñoz, E. (2001): Politica cientifica (y tecnológica) en España: Un siglo de intenciones, Ciencia al Día 4/1.

Natenzon, C. \& S. Funtowicz (2003): Ciencia, gobierno y participación ciudadana en: López Cerezo, J. A. \& A. Ibarra (eds). La democratización de la ciencia. San Sebastián: Cátedra Sánchez-Mazas.

Olazan, M. et al. (2004): ¿Hacia una segunda transición en la ciencia? Política cientifica y grupos de investigación, Revista de Sociologia Española, 4.

Petersen, J. (ed.) (1984): Citizen Participation in Science Policy. Amherst: University of Massachusetts Press.

PRICM (1990): I Plan regional de investigación de la Comunidad de Madrid 1990-1993.
PRICM (1994): I/ Plan regional de investigación de la Comunidad de Madrid 1994-1997. Madrid: Consejeria de Educación y Cultura.

PRICIT (1999): III Plan regional de investigación cientifica e innovación tecnológica de la Comunidad de Madrid 2000-2003. Madrid: Consejeria de Educación <htpp://www.dicm.csic.es/ pricit.pdf>

PRICIT (2005): IV Plan regional de investigación cientifica e innovación tecnológica de la Comunidad de Madrid, 2004-2008. Madrid: Dirección general de investigación. Consejería de Educación.

Sánchez Ron, J. M. (coord.) (1989): 19071987. La Junta para Ampliación de Estudios e Investigaciones Científicas 80 años después, Madrid: Consejo Superior de Investigaciones Científicas

Sanz Menéndez, L. \& M. J. Santesmases (1996): Ciencia y politica: interacciones entre el Estado y el sistema de investigación, Zona Abierta, 75/76.

Sanz Menéndez, L. (1997): Estado, ciencia y tecnología en España: 1939-1997. Madrid: Alianza.

Shapins, S. (2000): La revolución científica. Una interpretación alternativa. Barcelona: Paidós.

Todt, 0. (2003): Potencialidades y riesgos de la participación, en: López Cerezo, J.A. \& A. Ibarra (eds,), La democratización de la ciencia. San Sebastián: Cátedra Sánchez-Mazas.

Webler, T. \& S. Tuler (2002): Unlocking the Puzzle of Public Participation, Bulletin of Science, Technology \& Society, 22/3, 179-189. 